\title{
THE EQUILIBRIUM SHAPE OF A MISFITTING PRECIPITATE
}

\author{
M. E. THOMPSON, C. S. SU and P. W. VOORHEES \\ Department of Materials Science and Engineering, Northwestern University, Evanston, IL 60208, U.S.A.
}

(Received 16 July 1993)

\begin{abstract}
We examine the equilibrium morphologies of precipitates with either a tetragonal or purely dilatational misfit in an elastically anisotropic medium with cubic symmetry under conditions of plane strain. We find that particles with a dilatational misfit are nearly spherical at allomes, take on four-fold symmetric shapes at intermediate sizes and then undergo a supercritical symmetry-breaking bifurcation to two-fold symmetric shapes aligned along the elastically soft directions of the crystal. A tetragonal misfit breaks this four-fold to two-fold supercritical bifurcation when the direction of the tetragonality is coincident with one of the elastically soft directions of the crystal. Such a tetragonal misfit can lead to two-fold equilibrium particle shapes which are local energy minima, or metastable, and in some cases have large negative interfacial curvatures. When the tetragonality is not in an elastically soft direction, the supercritical bifurcation is not broken and the particles can take on unusual diamond-like or S-shaped morphologies.
\end{abstract}

\section{INTRODUCTION}

In the absuce of elastic stress, the factors influencing the equilibrium shape of a second-phase particle embedded in a matrix are well understood. In this case the equilibrium morphology of a particle is established solely by the interfacial energy and its dependence on crystallographic orientation. Specifically, the equilibrium shape can be found by minimizing the total interfacial energy subject to the constraint of constant particle volume. The properties of this variational problem have been explored quite thoroughly, and have been placed on a rigorous mathematical footing by Taylor [1] and Fonseca [2]. It is possible to snow that the particle shape which minimizes the total interfacial energy must be convex and is independent of the size of the particle [3,4]. In addition, for a given dependence of the interfacial energy on crystallographic orientation the particle shape which yields a global energy minimum is the one provided by the Wulff construction and it is unique [4], although there can be many interfacial energy functions which yield the same Wulff shape $[5,6]$.

In contrast, the influence of elastic stress on the equilibrium shape of a particle has not received as much attention and has been recently reviewed [7]. In the majority of the theoretical work on the effects of elastic stress on the shape of a misfitting particle it has been assumed that the particle shape will be that which minimizes the elastic energy within a fixed class of geometric shapes, usually ellipeoids, cubes or plates [8-13]. While these elastic energy minimization approaches have been successful in predicting such properties as the habit planes of plate-like precipitates [10], it is clear that the particle shapes observed experimentally can be quite different from any one of these shapes. Adding the interfacial energy to these elastic energy calculations provides a more realistic description of the particle shape. In this case the interfacial energy can establish the eccentricity of an ellipsoidal particle of a certain size $[14,15]$ or provide the possible sequence of particle shapes during particle splitting in elastically stressed solids [9]. These calculations have also shown that elastic stress can induce particle shape bifurcations $[14,15]$. The importance of this discovery will become clear in the work described herein. The major drawbock of these calculations, however, is that once the interfacial energy is added to the problem, these simple geometric shapes are no longer equilibrium shape; it can be shown that the chemical or diffusion potential is nonuniform in a system with such particles [16]. Thus, searching for an energy minimum within this restricted class of geometric shapes is, in essence, finding a form of constrained equilibrium. As the effects of these constraints are unclear, it is necessary to consider the equilibrium shape problem in the broader context of arbitrarily shaped particles.

Three approaches have been used in an effort to investigate the evolution of arbitrarily shaped misfitting particles: a diffuse interface model wherein a Cahn-Hilliard-like equation [17], or its discrete analogue [18], is used to describe the temporal evolution of the concentration field, a model which breaks a particle into elementary cubes and then considers the energy changes associated with mearrangements of these cubes $[19,20]$, and finally a 
continuum, sharp interface model where the particle evolves by diffusion of mass [21]. While most of these calculations have concentrated on the dynamics of the single or multi-particle evolution process, it is clear from each study that elastic stress can alter the equilibrium particle shape from that which minimizes the surface energy.

To further elucidate the effects of elastic stress on particle morphology, we have determined the equilibrium shape of particles with dilatational or tetragonal misfits in systems with the cubic anisotropic elastic constants of either nickel or zirconia. We have searched for particle shapes which guarantee that the diffusion potential in the system is uniform at all points, and thus do not constrain the particles to be within any class of simple geometric shapes. We employ bifurcation tracking methods to determine if the shapes are energy minima or maxima, as well as to ensure that we have located all of the connected families of equilibrium particle shapes for a given set of parameters. In addition, as the theory is framed in the context of macroscopically measurable quantities, it is possible to suggest a new method for determining the interfacial energy of a coherent interface which does not require measurement of the coarsening kinetics of a two-phase mixture.

\section{THERMODYNAMICS}

The variational problem governing the equilibrium shape of an isolated particle and the connection between this variational principle and the thermodynamics of stressed solids developed by others [22-26] has been established in the work of Gurtin and Voorhees [27]. Here we state the results of their work as it forms the basis of the numerical method we have employed to determine the equilibrium shape of a particle.

For small deviations about a natural state, the equilibrium state wherein the interface between the particle and matrix is flat and both phases are stress-free, it is reasonable to approximate the grand canonical free energy density of both matrix, $\alpha$, and particle, $\beta$, as a linear function of the deviation of the diffusion potential from that state and a quadratic function of the strain. Thus, the elastic energy density, $W\left(\mathbf{E}-\mathbf{E}_{0}\right)$, is given by

$$
W\left(\mathbf{E}-\mathbf{E}_{0}\right)=\frac{1}{2}\left(\mathbf{E}-\mathbf{E}_{0}\right) \cdot \mathbf{C}\left(\mathbf{E}-\mathbf{E}_{0}\right)
$$

and the following constitutive law for the stress, T, holds

$$
\mathbf{T}=\mathbf{C}\left(\mathbf{E}-\mathbf{E}_{0}\right)
$$

where $\mathbf{E}$ is the total strain tensor, $\mathbf{C}$ is the elastic constant tensor, and $E_{0}$ is the strain tensor of each phase relative to their natural states. Choosing stressfree $\alpha$ as the reference state for strain, $\mathbf{E}_{\mathbf{0}}$ is given by

$$
\mathbf{E}_{0}(\mathbf{x})= \begin{cases}\mathbf{O} & \mathbf{x} \text { in } \alpha \\ \mathbf{E}^{\mathrm{T}} & \mathbf{x} \text { in } \beta\end{cases}
$$

$\mathbf{E}^{\mathbf{T}}$ is the stress-free transformation strain or eigenstrain, and $\mathbf{x}$ is a position vector.

Under this choice for the grud canonical fue energy, the lattice parameter of each phase is not a function of composition, and by neglecting the interfacial excess stress, the equilibrium shape of a particle is given by an extremum in the total energy of the system, $\mathscr{E}$, where

$$
\mathscr{E}=\int_{\Omega}\left[W\left(\mathbf{E}-\mathbf{E}_{0}\right)-\mathbf{T}^{*} \cdot \mathbf{E}\right] \mathrm{d} V+\int_{S} \sigma(\mathbf{n}) \mathrm{d} A
$$

along with the requirements that,

$$
\begin{gathered}
M=\text { constant in } \Omega \\
\operatorname{div} \mathrm{T}=0
\end{gathered}
$$

and

$$
V^{\beta}=\text { constant }
$$

where $\Omega$ is the two-phase system which is composed of an infinite matrix of $\alpha$ and a single precipitate of $\beta, T^{*}$ is the applied stress on the boundary of $\Omega, S$ is the surface of the particle, $\sigma(\mathbf{n})$ is the interfacial energy, $\mathbf{n}$ is the normal to the particle interface pointing from $\beta$ to $\alpha, M$ is difference between the value of the diffusion potential in the system with the misfitting particle and the constant value of the diffusion potential in the natural state and $V^{B}$ is the volume of the particle. The first two requirements are simply those of chemical and elastic equilibrium, respectively.

The value of the constant diffusion potential present in a system which extremizes the energy $\mathscr{E}$ is given by the value of $M$ at the particle-matrix interface [27],

$$
\Lambda M=\llbracket W\left(\mathbf{E}-\mathbf{E}_{0}\right) \rrbracket-\llbracket \mathbf{T} \cdot \mathbf{E} \rrbracket+\operatorname{div}_{s} \boldsymbol{\xi}
$$

along with the constraint equation (2.7), where $\Lambda=\rho^{\beta}-\rho^{x}, \rho$ is the mass density in the designated phase, $\llbracket \phi \rrbracket=\phi^{\beta}-\phi^{\alpha}$ for a quantity $\phi$ at the interface, $\operatorname{div}_{\mathbf{s}}$ is the surface divergence operator, and $\xi$ is the $\xi$-vector of Cahn and Hoffman $[28,29]$. Since all of the quantities appearing in equation (2.8) are evaluated at the interface, equation (2.8) with $M$ chosen to be a constant can be used to derive an equation for the equilibrium shape.

\section{AN APPLICATION OF THE THERMODYNAMICS}

We shall consider a system with a cubic elastio misotropy, equal elastic constants between the article and matrix, no externally applied stress, and an isotropic interfacial energy. The particle will be taken as infinite in the [001] direction such that the system is in a state of plane strain. The only source of stress will be the stress-free transformation strain of magnitude $\epsilon$ and $\epsilon^{\prime}$ in the [100] and [010] directions, respectively. Therefore $\mathbf{E}^{\mathrm{T}}$ is given by

$$
\mathbf{E}^{\mathrm{T}}=\left(\begin{array}{ccc}
E & 0 & 0 \\
0 & \epsilon^{2} & 0 \\
0 & 0 & 0
\end{array}\right)
$$

For a system with a purely dilatational misfit $\epsilon^{\prime}=\epsilon$. 


\subsection{The equation for the equilibrium shape}

As we are interested in the effects of elastic stress on the equilibrium shape of a particle, it is advantageous to choose energy and length scales which allow the equilibrium shape of a body to be computed easily in the absence of stress. We will thus scale the energy of the system by a characteristic interfacial energy, $\sigma l^{2}$, where $l$ is the characteristic size of a particle defined by the relationship $a^{\beta}=A^{\beta} / l^{2}, A^{\beta}$ is the dimensional area of the particle in the $(001)$ plane, and $a^{\beta}$ is the dimensionless area of the particle which is chosen to be $\pi$. The stress will be scaled by $C_{44} \epsilon$, where $C_{44}$ is one of the three independent elastic constants in a cubic system, and the strains will be scaled by $\epsilon$. These scalings for stress and strain imply that $C_{44} \epsilon^{2}$ scales the elastic energy density.

Using these scalings and aforementioned assumptions, equation (2.4) becomes

$$
e=L \int_{\omega} w\left(\mathrm{e}-\mathrm{e}_{0}\right) \mathrm{d} a+s_{\mathrm{t}}
$$

where $e=\varnothing /\left(\sigma l^{2}\right), w=W /\left(C_{44} \epsilon^{2}\right), \mathbf{e}=\mathbf{E} / \epsilon, \mathbf{e}_{0}=\mathbf{E}_{0} / \epsilon$, $\omega$ is the dimensionless cross-sectional area of the system in the (001) plane, $a=A / l^{2}, s_{\mathrm{t}}$ is the dimensionless total arc length of the particle in the (001) plane and $L=\epsilon^{2} C_{44} l / \sigma$. The stress-free transformation strain in units of $\epsilon$ becomes

$$
\mathbf{e}^{\mathrm{T}}=\left(\begin{array}{lll}
1 & 0 & 0 \\
0 & \epsilon^{\mathrm{S}} & 0 \\
0 & 0 & 0
\end{array}\right)
$$

where $\epsilon^{\mathrm{r}}=\epsilon^{\mathrm{t}} / \epsilon$. The integral in equation (2.4) over the surface of the particle becomes simply the total arc length of a particle because we have assumed that the interfacial energy is independent of orientation and the particle is infinite in the [001] direction. Due to the plane strain nature of the elastic field, only an integration over the $(001)$ plane is necessary to determine the elastic energy. The parameter $L$ indicates the relative contributions of elastic and interfacial energy to the total energy of the system. As $L \propto l$ it is clear that as the particle increases in size, the effects of the elastic stress on the equilibrium shape become more important. However, as $L$ is composed of a collection of materials parameters more than simply the size of a particle is crucial in determining the influence of elastic energy.

The equation for the diffusion potential at the interface, equation (2.8), becomes

$$
\left.m=L\left\{\llbracket w\left(\mathbf{e}-\mathbf{e}_{0}\right)\right]-\llbracket \mathbf{t} \cdot \mathbf{e} \rrbracket\right\}+\kappa
$$

where $m$ is the dimensionless diffusion potential, $m=M \Lambda l / \sigma$, and $\kappa$ is the dimensionless mean curvature of the interface taken to be positive for a spherical particle of $\beta$. In deriving equation (3.4) we have used the result that $\operatorname{div}_{\mathrm{s}} \xi=\sigma \mathscr{K}$, where $\mathscr{K}$ is the dimensional mean curvature, when the interfacial energy is isotropic. The value of the constant diffusion potential for an energy-extremizing shape is set by the magnitude of the dimensionless area of the particle.

The expression for the diffusion potential at the interface can be used to derive an equation for the equilibrium shape of a particle only if the strain in the $\alpha$ and $\beta$ phases at the interface can be expressed as a function of the shape of the particle. This requires a method of solving for the elastic field surrounding an arbitrarily shaped misfitting particle in an elastically anisotropic medium that does not require integrations over the bulk phases. Thus, the objective is to map the entire elastic problem onto the interface of the particle. A method to accomplish this has been developed previously [21] and here we state only the key results and modifications of the approach necessary to determine the interfacial stress field for a tetragonally misfitting particle.

The elastic field at any point in the system can be represented as an integral only over the interface [30]

$$
u_{j, k}(\mathbf{x})=\left(c_{i 11}+c_{i 222} \epsilon^{\mathrm{r}}\right) \int_{s} g_{i j, k}\left(\mathbf{x}, \mathbf{x}^{\prime}\right) n_{i}^{\prime} \mathrm{d} s^{\prime}
$$

where $s^{\prime}$ is the arc length along the interface, $g_{V}$ are the dimensionless components of the elastic Green's function tensor, $\mathbf{c}=\mathbf{C} / C_{\mathbf{4}}, u_{j}$ are the dimensionless components of the displacement vector measured with respect to the reference state of stress-free $\alpha$, and $x$ and $x^{\prime}\left(s^{\prime}\right)$ locate field and interfacial points, respectively, in the $\left(x_{1}, x_{2}\right)$, or (001), plane. Summation over repeated indices from 1 to 3 is assumed and the commas denote partial differentiations with respect to the noted index. The elastic field and the total strain field follow from the definitions given in the previous section and the relation

$$
e_{i j}=\left(u_{i, j}+u_{j,}\right) / 2 .
$$

To avoid using numerical quadratures or Fourier transforms to evaluate the Green's function at each point on the interface, we employ an effectively analytic form for the plane strain Green's function developed by Stroh [21, 31].

The value of the displacement gradient at the particle-matrix interface in the $\alpha$ phase, $u_{j, n}^{\alpha}$, and in the $\beta$ phase, $u_{j, n}^{\beta}$, at a point $s^{0}$ can be found by talking the limit $x \rightarrow x^{\prime}$ in equation (3.5). Following the method described previously [21], the displacement gradients are given by

$$
u_{j, n}^{\alpha}=-\llbracket u_{j, n} \rrbracket / 2+p_{j n}, \quad u_{j, n}^{\beta}=\llbracket u_{j, n} \rrbracket / 2+p_{j n}
$$

where

$$
\begin{aligned}
p_{j n}= & -\frac{1}{\pi} \sum_{a=1}^{2} \\
& \times \int_{s} \frac{\left(x_{1}-x_{1}^{\prime}\right) \mathcal{O}_{j n}^{a}+\left(x_{2}-x_{2}^{\prime}\right) P_{j n}^{u}}{\left[\left(x_{1}-x_{1}^{\prime}\right)+P_{a}^{\mathrm{R}}\left(x_{2}-x_{2}^{\prime}\right)\right]^{2}+\left[P_{a}^{1}\left(x_{2}-x_{2}^{\prime}\right)\right]^{2}} d s^{\prime}
\end{aligned}
$$


and

$$
\begin{aligned}
\mathscr{O}_{j n}^{a}= & B_{j n}^{a} x_{2, s}^{\prime}-D_{j n}^{a} x_{1, s}^{\prime} \\
\mathscr{P}_{j n}^{a}= & C_{j n}^{a} x_{2, s}^{\prime}-E_{j n}^{a} x_{1, s}^{\prime} \\
B_{j n}^{a}= & \left(c_{11}+\epsilon^{\mathrm{I}} c_{12}\right)\left[F_{\mathrm{l}}^{a} P_{a}^{1} \delta_{2 n}+G_{j,}^{a}\left(\delta_{1 n}+P_{a}^{\mathrm{R}} \delta_{2 n}\right)\right] \\
C_{j n}^{a}= & \left(c_{11}+\epsilon^{\mathrm{r}} c_{12}\right)\left[-F_{1 j}^{a} P_{a}^{1} \delta_{1 n}+G_{1 j}^{a}\left(P_{a}^{\mathrm{R}} \delta_{1 n}\right.\right. \\
& \left.\left.+\left|P_{a}\right|^{2} \delta_{2 n}\right)\right] \\
D_{j n}^{a}= & \left(c_{12}+\epsilon^{\mathrm{r}} c_{11}\right)\left[F_{2 j}^{a} P_{a}^{1} \delta_{2 n}+G_{2 j}^{a}\left(\delta_{1 n}+P_{a}^{\mathrm{R}} \delta_{2 n}\right)\right] \\
E_{j n}^{a}= & \left(c_{12}+\epsilon^{\mathrm{r}} c_{11}\right)\left[-F_{2 j}^{a} P_{a}^{1} \delta_{1 n}+G_{2 j}^{a}\left(P_{a}^{\mathrm{R}} \delta_{1 n}\right.\right. \\
& \left.\left.+\left|P_{a}\right|^{2} \delta_{2 n}\right)\right] \\
F_{i j}^{a}= & A_{i a}^{\mathrm{R}} A_{j a}^{\mathrm{R}}-A_{i a}^{\mathrm{I}} A_{j a}^{\mathrm{l}} \\
G_{i j}^{a}= & A_{i a}^{l} A_{j a}^{\mathrm{R}}-A_{i a}^{\mathrm{R}} A_{j a}^{\mathrm{I}}
\end{aligned}
$$

and the superscripts I, $\mathbf{R}$ denote imaginary and real parts, respectively. The components of the matrices $B_{j n}^{a}, C_{j n}^{a}, D_{j n}^{a}, E_{j n}^{a}$, the vectors $A_{i a}^{1} A_{j a}^{\mathrm{R}}$ and the constants $P_{a}^{1}, P_{a}^{\mathbf{R}}$ need only be computed once for a given anisotropy ratio $A_{\mathrm{r}}$, where $A_{\mathrm{r}}=2 c_{44} /\left(c_{11}-c_{12}\right)$ [21]. In equation (3.7) both $x$ and $x^{\prime}$ are on the interface and thus the integral must be interpreted as a principle value integral. The jump in the displacement gradient is given by

$$
\llbracket u_{j, n} \rrbracket=-\sum_{a=1}^{2} \frac{2 \pi\left(N_{j n}^{a} \mathscr{C}^{a}-M_{j n}^{a} \mathscr{C}^{a}\right)}{\mathscr{C}^{a}\left[\mathscr{A}^{a} \mathscr{C}^{a}-\left(\mathscr{O}^{a}\right)^{2}\right]^{1 / 2}}
$$

where

$$
\begin{aligned}
N_{j n}^{a}= & x_{2, s}^{0}\left(B_{j n}^{a} x_{2, s}^{0}-D_{j n}^{a} x_{1, s}^{0}\right)-x_{1, s}^{0}\left(C_{j n}^{a} x_{2, s}^{0}\right. \\
& \left.-E_{j n}^{a} x_{1, s}^{0}\right) \\
M_{j n}^{a}= & -x_{1, s}^{0}\left(B_{j n}^{a} x_{2, s}^{0}-D_{j n}^{a} x_{1, s}^{0}\right) \\
& -x_{2, s}^{0}\left(C_{j n}^{a} x_{2, s}^{0}-E_{j n}^{a} x_{1, s}^{0}\right) \\
\mathscr{A}^{a}= & \left(x_{2, s}^{0}\right)^{2}-2 P_{a}^{\mathrm{R}} x_{2, s}^{0} x_{1, s}^{0}+\left|P_{a}\right|^{2}\left(x_{1, s}^{0}\right)^{2} \\
\mathscr{P}^{a}= & -x_{2, s}^{0}\left(x_{1, s}^{0}+P_{a}^{\mathrm{R}} x_{2, s}^{0}\right) \\
& +x_{1, s}^{0}\left(P_{a}^{\mathrm{R}} x_{1, s}^{0}+\left|P_{a}\right|^{2} x_{2, s}^{0}\right) \\
\mathscr{C}^{a}= & \left(x_{1, s}^{0}\right)^{2}+2 P_{a}^{\mathrm{R}} x_{1, s}^{0} x_{2, s}^{0}+\left|P_{a}\right|^{2}\left(x_{2, s}^{0}\right)^{2}
\end{aligned}
$$

and the superscript 0 denotes the values of the quantities at $s^{0}$.

Using this form for the displacement gradient at the interface in equation (3.6), and using equation (2.2) in equation (3.4) yields an integrodifferential equation for the shape of a particle which extremizes 8

$$
\begin{aligned}
m= & \frac{1}{2} L c_{i j k l}\left\{-2 p_{i j}(s) e_{k l}^{\mathrm{T}}-\llbracket u_{i, j}(s) \rrbracket e_{k l}^{\mathrm{T}}\right. \\
& \left.+e_{i j}^{\mathrm{T}} e_{k l}^{\mathrm{T}}+\llbracket u_{i, j}(s) \rrbracket \llbracket u_{k, l}(s) \rrbracket\right\}+\kappa(s)
\end{aligned}
$$

where $\mathrm{e}^{\mathrm{T}}$ is the stress-free transformation strain given in equation (3.3) and $c$ is the cubically anisotropic elastic constant tensor. This equation must be solved subject to the constraint of constant particle area to determine the particle shape which extremizes the total energy of the system.

\subsection{The total energy of the system}

A direct evaluation of the total energy of the system requires a method to determine the total elastic energy of the system. A straightforward method for finding the total elastic energy of the system would be to evaluate numerically the following two dimensional integral

$$
e_{\mathrm{e}}=L \int_{\omega} w\left(\mathrm{e}-\mathrm{e}_{0}\right) \mathrm{d} a
$$

where $e_{\mathrm{e}}$ is the total elastic energy. Unfortunately, this is not computationally efficient and can be quite inaccurate due to the rapid change in the elastic fields very close to the particle-matrix interface. Thus, we modify a method due to Eshelby [32] which allows the total elastic energy of the system to be determined by performing integrations of the displacement gradients only over the interface of the particle. This is particularly advantageous because the integration will be one-dimensional over a periodic domain and thus we can use a spectrally accurate trapezoidal quadrature to evaluate the integral.

Eshelby showed that for a system consisting of an isolated particle in an infinite matrix with no applied stress at infinity equation (3.11) can be written as

$$
e_{e}=\frac{L}{2} \int_{s}\left(u_{i}^{\beta}-u_{i}^{\alpha}\right) T_{i j} n_{j} \mathrm{~d} s^{\prime}
$$

where $u_{i}^{\beta}$ and $u_{i}^{\alpha}$ are components of the elastic displacements at the interface measured relative to the natural states of each phase. Since the interface is coherent

$$
u_{i}^{\mathrm{T}}=u_{i}^{\beta}-u_{i}^{\alpha}
$$

where $u_{i}^{\top}$ are components of the transformation displacement. Using equation (3.13) in equation (3.12) yields

$$
e_{e}=\frac{L}{2} \int_{s} u_{i}^{\mathrm{T}} T_{v} n_{j} \mathrm{~d} s^{\prime}
$$

The transformation displacement for the transformation strain given in equation (3.3) is found by equating each diagonal component of the two tensors, equation (3.6) and (3.3), and integrating to obtain

$$
\begin{aligned}
& u_{1}^{\mathrm{T}}=x_{1}+f\left(x_{2}\right) \\
& u_{2}^{\mathrm{T}}=\epsilon^{\mathrm{T}} x_{2}+g\left(x_{1}\right)
\end{aligned}
$$

where $f\left(x_{2}\right)$ and $g\left(x_{1}\right)$ are unknown functions. Since $e_{i j}^{\top}=0$ for $i \neq j$ and using equation (3.15) we find

$$
\frac{\mathrm{d} f}{\mathrm{~d} x_{2}}+\frac{\mathrm{d} g}{\mathrm{~d} x_{1}}=0 \text {. }
$$

As neither rigid body translations nor rotations alter the elastic energy of the system, we assume, with no loss of generality, that $\boldsymbol{u}_{i, j}^{\mathrm{T}}-\boldsymbol{u}_{j, 1}^{\mathrm{T}}=0$ thus,

$$
\frac{\mathrm{d} f}{\mathrm{~d} x_{2}}-\frac{\mathrm{d} g}{\mathrm{~d} x_{1}}=0 \text {. }
$$


Combining equations (3.16) and (3.17) restricts $f$ and $g$ to being constants. As elasticity theory permits displacement components to vary by constant values without affecting either the stress and strain fields or the elastic energy, we take both constants $f$ and $g$ to be zero. Thus, the components of the transformation displacement are given by

$$
\begin{aligned}
& u_{1}^{\mathrm{T}}=x_{1} \\
& u_{2}^{\mathrm{T}}=\epsilon^{\mathrm{r}} x_{2} .
\end{aligned}
$$

Using equation (3.18) in equation (3.14) and the stress tensor calculated in the manner described above, it is possible to compute the total elastic energy of the system via one-dimensional integration.

\subsection{Numerical method}

Equation (3.10) is a nonlinear integrodifferential equation which, for $L \sim O(1)$, requires a numerical method to find a solution. As the only unknown in equation (3.10) is the shape of the interface $\mathrm{x}(s)$, the dimensionality of the problem has been effectively reduced by one. Therefore, it is possible to attain very good numerical resolution of the shape of the particle which extremizes the energy of the system by solving equation (3.10). In addition, we employ spectrally accurate interpolation and quadrature schemes to determine $m$ at a point on the interface. This scheme ensures that the error introduced by the discretization decreases exponentially with the number of points used along the interface.

We shall modify the spectral interpolation approach used by Brush et al. [33] to the case of a closed two-dimensional contour with continuous curvature as a function of the arc length $s$. We shall not make any assumption on the symmetry of the particle, and thus express the curvature of the particle as a general complex Fourier series of the form

$$
\kappa(\xi)=\sum_{n=-\infty}^{\infty} a_{n} \exp ^{-(2 \times i n \xi)}
$$

where $\xi=s / s_{\mathrm{T}}$ is the normalized arc length, and the Fourier coefficients, $a_{n}$, are complex with real and imaginary parts given by, $a_{n}^{R}$ and $a_{n}^{l}$ respectively. The tangent angle to the interface, $\phi(\xi)$, as measured from the $x_{1}$ axis, may be determined by integrating the following identity

$$
\frac{\mathrm{d} \phi(\xi)}{\mathrm{d} \xi}=s_{\mathrm{T}} \kappa(\xi)
$$

and using equation (3.19) along with the identity $a_{-n}=a_{n}^{*}$ for real quantities to yield,

$$
\begin{array}{r}
\phi(\xi)-\phi(0)=s_{\mathrm{T}} a_{0}^{\mathrm{R}} \xi+2 s_{\mathrm{T}} \sum_{n=1}^{\infty}\left\{\frac{a_{n}^{\mathrm{R}}}{2 \pi n} \sin 2 \pi n \xi\right. \\
\left.+\frac{a_{n}^{1}}{2 \pi n}(1-\cos 2 \pi n \xi)\right\} .
\end{array}
$$

The reference point on the contour from which arc length is measured will be taken as a point where the tangent angle is zero and thus $\phi(0)=0$. Further, the value of the tangent angle is taken to range from 0 to $2 \pi$ so that, for a closed contour, $\phi(1)=\phi(0)+2 \pi$. Applying this relation to equation (3.21) gives the following

$$
s_{\mathrm{T}}=\frac{2 \pi}{a_{0}^{\mathrm{R}}}
$$

which determines the total arc length of the contour in terms of the initial Fourier coefficient in the expansion for the interfacial curvature. As $\phi(\xi)$ can be determined from $a_{n}, \cos \phi(\xi)$ and $\sin \phi(\xi)$ can also be represented as a Fourier series

$$
\cos \phi(\xi)=\sum_{n=-\infty}^{\infty} b_{n} \exp ^{(-2 \pi i n \xi)}
$$

and

$$
\sin \phi(\xi)=\sum_{n=-\infty}^{\infty} c_{n} \exp ^{(-2 \pi i n \xi)}
$$

where the coefficients are determined by a fast Fourier transform, $b_{n}$ are complex with real and imaginary parts, $b_{n}^{\mathrm{R}}$ and $b_{n}^{1}$ respectively, and $c_{n}$ are also complex with real and imaginary parts $c_{n}^{R}$ and $c_{n}^{1}$ respectively. Using equations (3.23) and (3.24) in the following

$$
\begin{aligned}
& \frac{\mathrm{d} x(\xi)}{\mathrm{d} \xi}=s_{\mathrm{T}} \cos \phi(\xi) \\
& \frac{\mathrm{d} y(\xi)}{\mathrm{d} \xi}=s_{\mathrm{T}} \sin \phi(\xi)
\end{aligned}
$$

and integrating the resulting expressions yields the coordinates of the interface

$$
\begin{aligned}
& x(\xi)=s_{\mathrm{T}} b_{0}^{\mathrm{R}} \xi+2 s_{\mathrm{T}} \sum_{n=1}^{\infty}\left\{\frac{b_{n}^{\mathrm{R}}}{2 \pi n} \sin 2 \pi n \xi\right. \\
&\left.+\frac{b_{n}^{1}}{2 \pi n}(1-\cos 2 \pi n \xi)\right\} \\
& y(\xi)=s_{\mathrm{T}} c_{0}^{\mathrm{R}} \xi+2 s_{\mathrm{T}} \sum_{n=1}^{\infty}\left\{\frac{c_{n}^{\mathrm{R}}}{2 \pi n} \sin 2 \pi n \xi\right. \\
&\left.+\frac{c_{n}^{1}}{2 \pi n}(1-\cos 2 \pi n \xi)\right\}
\end{aligned}
$$

where $x(0)$ and $y(0)$ are set to zero. This selection of $x(0)$ and $y(0)$ is valid as it simply places the origin of the coordinate system at a point on the contour where $\phi(0)=0$.

The energy extremizing particle shape is determined by finding that shape which gives a constant diffusion potential in the system. The value of this constant dimensionless diffusion potential, for a particular particle shape, is a function of the dimensionless particle size. Thus, it is also necessary to determine the dimensionless area of the particle, $a^{b}$, from an interpolation of the interfacial curvature as a function of arc length, i.e.

$$
a^{\beta}=\int_{0}^{1} x(\xi) \frac{\mathrm{d} y(\xi)}{\mathrm{d} \xi} \mathrm{d} \xi
$$


which, upon inserting equation (3.27) and the derivative of equation (3.28) with respect to $\xi$ and integrating, gives

$a^{A}=\frac{\mathrm{I}}{2} s_{\mathrm{T}}^{2} b_{0}^{\mathrm{R}} c_{0}^{\mathrm{R}}+2 s_{\mathrm{T}}^{2} \sum_{n=1}^{\infty}\left\{\frac{b_{n}^{1}\left(c_{n}^{\mathrm{R}}-c_{0}^{\mathrm{R}}\right)+c_{n}^{1}\left(b_{n}^{\mathrm{R}}-b_{n}^{\mathrm{R}}\right)}{2 \pi n}\right\}$.

Thus the area of the particle may be determined to the same accuracy as the interfacial coordinate representation without resorting to a numerical integration scheme over discrete points.

The above spectral interpolation scheme provides the curvature of the interface and the coordinates of the interface as a function of the arc length. Given the shape of the particle, the remaining quantities to be computed are $p_{i j}$. These principal value integrals are calculated using a trapezoidal rule over an alternating mesh. Using this technique it is possible to straddle the Cauchy singularity in the integrand and still maintain a spectral convergence rate $[21,34,35]$.

To determine the shape which extremizes the energy of the system numerically, the integrodifferential equation specifying the diffusion potential is evaluated at $N$ equally spaced points in arclength along the interface. This yields $N$ nonlinear equations for $m$ at each point. The infinite series defining the mean curvature is then truncated at $n=N / 2$ yielding $N$ Fourier coefficients to be determined, $a_{0}^{\mathrm{R}}, \ldots, a_{N / 2}^{\mathrm{R}}, a_{1}^{1}, \ldots, a_{n / 2-1}^{1}$. The values of $a_{0}^{1}$ and $a_{N / 2}^{1}$ are zero as the curvature is a real quantity. These $N$ Fourier coefficients form a vector, a, which, upon solution of the nonlinear system, gives the energy extremizing shape. In addition to the $N$ unknown Fourier coefficients, the value of the constant diffusion potential is an unknown, and thus we augment the $N$ nonlinear diffusion potential equations by the area constraint, equation (3.30). This $(N+1) \times(N+1)$ nonlinear system is then solved using Newton's method to give the shape which yields a constant value of $m$ at each point on the interface.

\subsection{Continuation, bifurcations and stability}

We follow a particular solution family by using the particle shape at a lower value of the parameter of interest, usually $L$, as an initial guess for the Newton's method program. This method of continuation may be used to map an entire solution family as a function of a system parameter. For a given particle shape denoted by a set of $N$ Fourier coefficients, a, we evaluate the diffusion potential at each point on the interface, as given by equation (3.10), and construct the following system of equations,

$$
\begin{gathered}
m_{i}(\mathbf{a})-m=\mathscr{F}_{j}(\mathbf{a}, m), \quad j=1, \ldots, N \\
a^{\beta}(\mathbf{a})-a_{0}^{\beta}=\mathscr{F}_{N+1}(\mathbf{a}, m)
\end{gathered}
$$

where $m_{i}$ is the diffusion potential at point $i$ on the interface, $m$ is the constant diffusion potential as determined by the area constraint, $F_{i}$ is a function which measures the deviation of $m_{i}$ from the equi- librium value, and $a_{0}^{\beta}$ is the constant area of $\beta$ which is chosen to be $\pi$. Thus we search for energy extremizing shapes characterized by the solution vectors, $\boldsymbol{\alpha}_{e}$, where,

$$
a_{e}=\left(\begin{array}{c}
a_{c} \\
m_{e}
\end{array}\right)
$$

such that equation (3.31) takes the form

$$
F_{i}\left(\alpha_{\mathrm{e}}\right)=0
$$

where $i=1, \ldots, N+1$.

If two solution families intersect for a given value of $L$ then slightly away from this point there must exist two solutions, $\alpha_{e}^{1}$ and $\alpha_{e}^{2}$. In the region near the intersection, the value of $\mathscr{F}_{(}\left(\alpha_{e}^{2}\right)$ may be approximated as

$$
\mathscr{F}_{i}\left(\alpha_{e}^{2}\right)=F_{i}\left(\alpha_{e}^{1}\right)+\left[\frac{\partial F_{i}}{\partial \alpha}\right]_{e=\alpha)}\left(\alpha_{e}^{2}-\alpha_{e}^{1}\right) \text {. }
$$

Applying equation (3.33) to (3.34) yields the following expression which specifies the condition necessary for the intersection of two solution families

$$
\left[\frac{\partial F_{1}}{\partial \alpha}\right]_{a=\alpha_{e}^{!}}\left(\alpha_{e}^{2}-\alpha_{e}^{l}\right)=0 .
$$

Neglecting the trivial solution, $\alpha_{e}^{l}=\alpha_{e}^{2}$, this equation is satisfied if and only if the determinant of the Jacobian matrix equals zero. This requires that an eigenvalue of the matrix is also zero and the corresponding eigenvector, $\bar{\alpha}$, is simply given by $\bar{\alpha}=\alpha_{e}^{2}-\alpha_{e}$.

While tracking a solution family, the eigenvalues of the Jacobian of the nonlinear system are monitored as $L$ is varied. If an eigenvalue passes through zero, a bifurcation point or a turning point has been encountered and there will be two or more shapes satisfying equation (3.33) at a given value of $L$. If the eigenvector corresponding to the zero eigenvalue possesses the same symmetry as the initial solution family, then a turning point has been reached. A bifurcation point is identified by an eigenvector possessing a different symmetry than the initial solution. The new solution family can be tracked by perturbing a member of the initial solution family at a slightly higher or lower value of $L$ with the eigenvector corresponding to the eigenvalue which has passed through zero. Thus for $L$ near the bifurcation point we write

$$
\boldsymbol{\alpha}_{\mathrm{e}}^{2}=\boldsymbol{\alpha}_{\mathrm{e}}^{1}+\epsilon \tilde{\boldsymbol{\alpha}}
$$

where $\alpha_{c}^{2}$ is the vector defining the new shape which is present past the bifurcation point, $\alpha_{\mathrm{c}}^{1}$ is the vector defining the initial shape, and $\epsilon$ is a small parameter, typically chosen to be $\pm 10^{-2}$. Since the nonlinear system is not based upon an expression for the first derivative of the energy of the system, the signs of the eigenvalues do not indicate if a solution is an energy minimum or maximum. Thus, to determine the stability of each solution family, we must employ a different technique.

The total energy of the system is given by equation (3.2) and is a function of the vector, $\boldsymbol{\alpha}$. As the particle is constrained to be of constant area, the morphologies which extremize the total energy must be 
restricted to the level set for which $a^{\beta}=a_{0}^{\beta}$. This is accomplished by defining a new energy, $h$, which is a Legendre transform of $e$

$$
h(\alpha)=e(\mathbf{a})-m\left[a^{\beta}(\mathbf{a})-a_{0}^{\beta}\right]
$$

where the variable conjugate to the area is the diffusion potential $m$. Particle shapes which extremize the energy thus satisfy the system

$$
\frac{\partial h}{\partial \alpha_{i}}=0
$$

for $i=1, \ldots, N+1$. The set of equations represented by equation (3.38) may be used to determine the particle shape and diffusion potential which extremizes the energy of the system. However, the method described previously, involving the solution of equation (3.10), is preferred as equation (3.38) requires many numerical evaluations of secondderivatives of the energy in order to solve the system. Equation (3.38) is useful, however, in determining the stability of the solutions provided by equation (3.10). The stability of the extrema may be determined from the eigenvalues of the Hessian matrix resulting from the second derivative of the energy, $h(\mathbf{a}, m)$. Using equation (3.37), the Hessian matrix, $H_{i j}$, is given by

$$
H_{i j}=\left(\frac{\partial^{2} h}{\partial \alpha_{i} \partial \alpha_{j}}\right) \text {. }
$$

The eigenvectors corresponding to positive eigenvalues of $H_{i j}$ denote perturbations which lead to an increase in system energy while eigenvectors corresponding to negative eigenvalues represent perturbations which reduce the total energy of the system. Thus, we can only determine relative energy extrema through this approach. If multiple energy minima are present, the absolute energy minimum can be determined simply by calculating $e$ directly for each $\boldsymbol{a}_{e}$.

\section{RESULTS}

In all of the results to follow the sides of the boxes used to display the equilibrium shapes will always be oriented such that they are along the [100] and [010] directions. In all of the bifurcation diagrams, dashed lines will denote energy maxima and solid lines will indicate energy minima. Occasionally, an eigenvalue would pass through zero whose eigenvector would correspond to an interface which was not closed. This is possible as certain combinations of the Fourier coefficients used in the expansion for the curvature produce open bodies. As these shapes are simply a result of our interpolation scheme (they disappeared when a cubic spline interpolation was used in the calculation) we ignored such bifurcations. In addition, a positive eigenvalue on the order of the numerical accuracy was frequently present which corresponded to an eigenvector possessing only an $a_{N / 2}^{\mathrm{R}}$ term. This eigenvector yields a perturbation of the form $\cos (n \pi)$, where $n$ is the integer denoting the mesh point of interest. This is an instability on the scale of the mesh and is the well-known sawtooth instability common to numerical boundary integral methods [36]. As this instability is numerical in nature, we neglected this positive eigenvalue.

\section{1. $A_{r}>1, \epsilon^{\prime}=1$}

Here we consider a system with the elastic anisotropy ratio of $\mathrm{Ni}, A_{\mathrm{r}}=2.52$. Thus, the elastically soft directions of the crystal are $\langle 100\rangle$. The precipitate has a purely dilatational misfit with the matrix.

The equilibrium shape at $L=0$ is a circle. If we use this shape as an initial guess for a nonzero value of $L$ we can access the family of four-fold symmetric shapes shown in Fig. 1. Evident is the progression of particle shapes as $L$ increases from the interfacial energy minimizing circle to square-like shapes which reflect the four-fold anisotropy of the elastic constants. The particles have regions of high curvature along the elastically hard $\langle 110\rangle$ directions and regions of low curvature along the $\langle 100\rangle$, where \langle\rangle denotes all the symmetry-equivalent crystallographic directions in the (001) plane. For $L \geq 25$ the curvature of the interface is negative near the centers of the particle sides. Since $L \sim l$, as a particle increases its size, for a given two-phase system, the energy extremizing shape will change. To illustrate this size dependence of the four-fold symmetric energy extremizing shapes, Fig. 2 shows nondimensionalized versions of these energy extremizing shapes for various $L$, relative to the size of a particle with $L=1$. Thus, one might expect to find that the shape of a particle will change smoothly along this family of four-fold symmetric shapes as its size increases.

However, as $L$ increases an eigenvalue of the Jacobian passes through zero at $L=5.6$. Above this

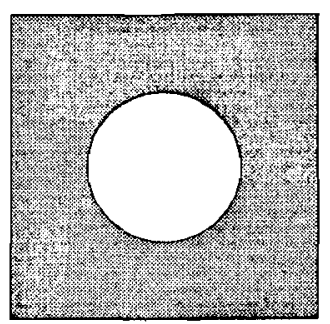

(a)

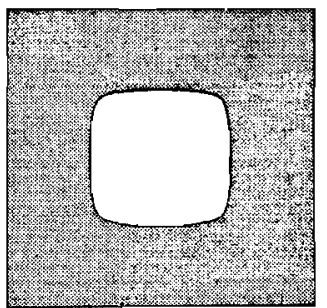

(c)

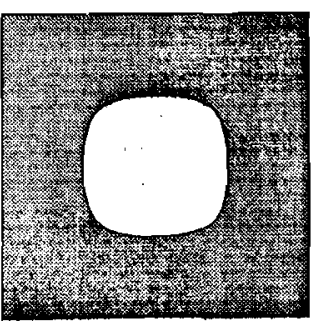

(b)

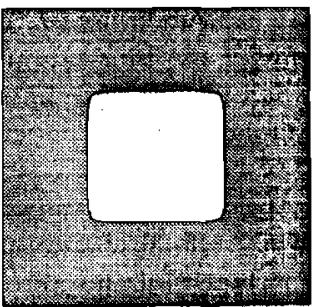

(d)
Fig. 1. Energy extremizing particle shapes for a misfitting particle with a purely dilatational misfit in a Ni matrix for various values of $L$ : (a) 0 , (b) 4 , (c) 10 , (d) 26 . 


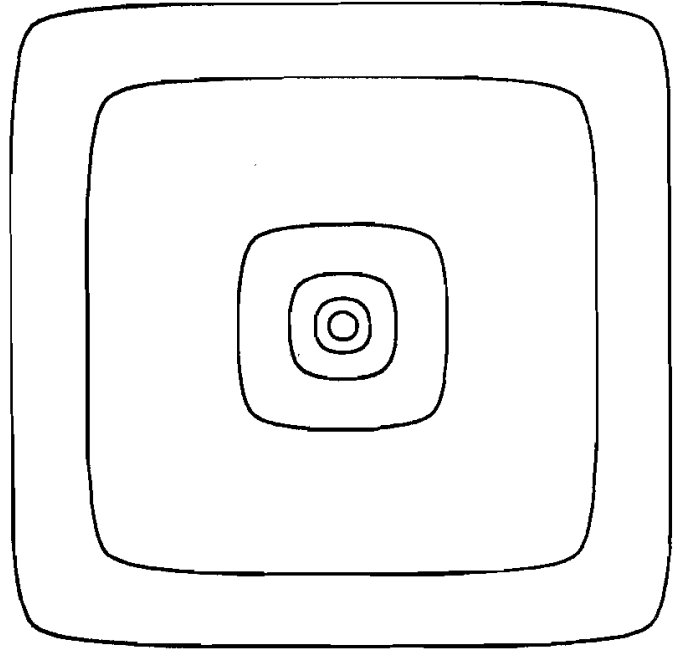

Fig. 2. Energy extremizing particle shapes for $L=1,2,4,8$, 20,26 , relative to the size of a particle with $L=1$.

critical value of $L$ we find the two-fold symmetric shapes shown in Fig. 3. In this case the long axis of the particles are aligned along the elastically soft [100] direction. However, if slightly past the bifurcation point we change the sign of $\epsilon$ in equation (3.36) we find two-fold shapes oriented along the [010] direction. These two-fold particle shapes no longer conform to the four-fold anisotropy of the elastic constants. While they possess the symmetry of a plate, they are definitely not plates as the curvature of the sides of the particles is positive and the corners are rounded. This reflects the influence of the interfacial energy on the equilibrium shape. However, as $L$ increases, the equilibrium shapes appear to be approaching the elastic energy minimizing shape of an infinitely long, infinitesimally thin plate oriented along one of the elastically soft $\langle 100\rangle$ directions $[26,37]$.

The passage of an eigenvalue through zero denotes the possibility of a shape bifurcation. By computing the eigenvalues of the Hessian at various values of $L$ for the energy extremizing four-fold shapes, we find that four-fold shapes are energy minima for $L<5.6$ and energy maxima for $L>5.6$. A similar analysis shows that the two-fold shapes are energy minima for $L>5.6$. We can classify each family of solutions by its value of $a_{2}^{R}$, one component of the vector $a$ of Fourier coefficients which define a particle shape. By plotting $a_{2}^{\mathrm{R}}$ as a function of $L$ for both families of solutions we obtain the bifurcation diagram shown in Fig. 4. In addition, we find that four-fold shapes are only unstable to two-fold perturbations in their shape for $5.6<L<26$, indicating that these four-fold shapes are stable with respect to interfacial perturbations which would lead to splitting.

The total interfacial energy of the four-fold symmetric shapes is clearly lower than that of the two-fold symmetric shapes, see the particles at $L=10$ in Fig. 4. However, the two-fold shapes are the total energy minimum. Thus, the elastic energy must be

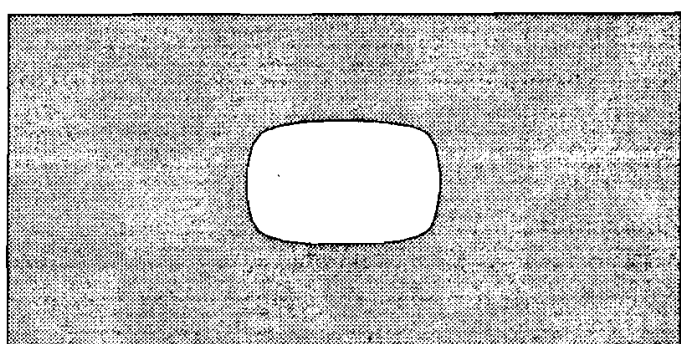

(a)

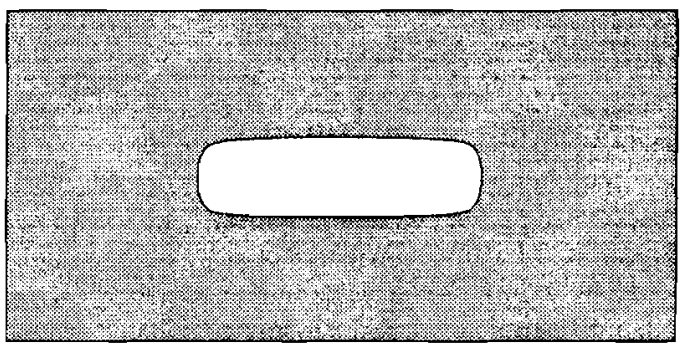

(b)

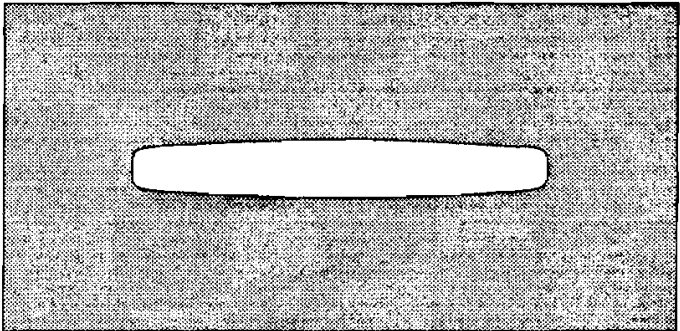

(c)

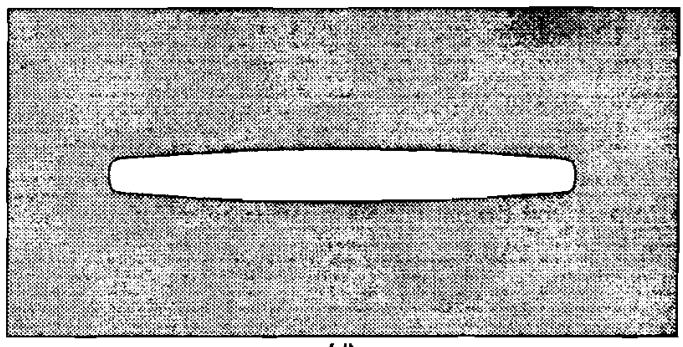

(d)

Fig. 3. Two-fold symmetric equilibrium shapes for a particle with a purely dilatational misfit in a Ni matrix for various values of $L$ : (a) 6, (b) 10, (c) 20, (d) 26 .

playing an important role in selecting the equilibrium shape. To investigate the reason behind the appearance of the symmetry breaking two-fold shapes we examine the total elastic energy density as a function of position. It is also instructive to decompose the elastic energy density, $w$, into its deviatoric, $w_{\mathrm{d}}$, and hydrostatic, $w_{h}$, components given by,

$$
\begin{aligned}
& w_{\mathrm{d}}=\frac{1}{2} \mathbf{t}^{\prime} \mathbf{e}^{\prime} \\
& w_{\mathrm{h}}=\frac{3}{2} p d
\end{aligned}
$$

where $t^{\prime}$ and $e^{\prime}$ are the deviatoric stress and strain tensors, respectively, $p$ is the hydrostatic pressure, and $d$ is the hydrostatic strain. 


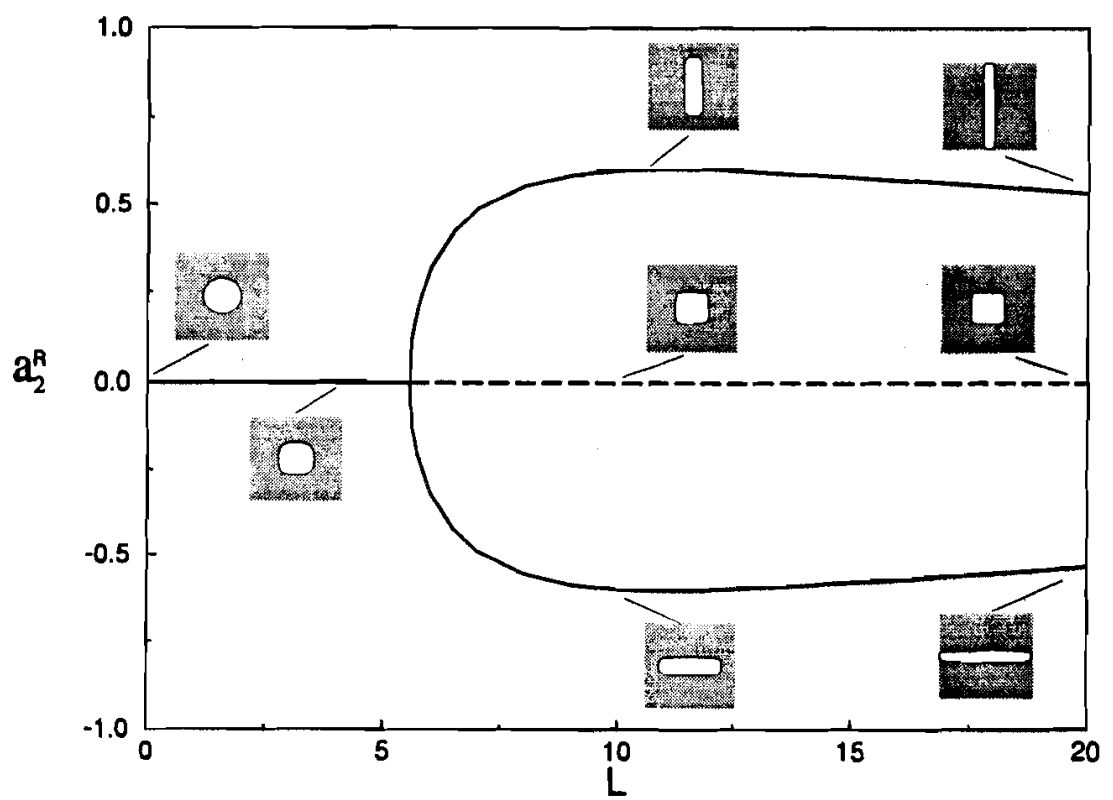

Fig. 4. Bifurcation diagram showing the supercritical bifurcation from four-fold symmetric shapes to two-fold symmetric shapes at $L=5.6$.

Thus the elastic energy density, $w$, is given by the sum of $w_{d}$ and $w_{h}$, each of which reflects different types of deformation. $w_{\mathrm{h}}$ measures the energy required to dilate the lattice while $w_{\mathrm{d}}$ measures the energy required to alter the shape of the lattice, since $\operatorname{tr}\left(\mathrm{e}^{\prime}\right)=0$. Figures 5 and 6 show $w, w_{\mathrm{d}}$, and $w_{\mathrm{h}}$ as functions of position in the [100] direction along the lines illustrated for the four-fold and two-fold shapes, respectively. In both cases, the matrix is in a state of nearly pure distortion, and the hydrostatic energy
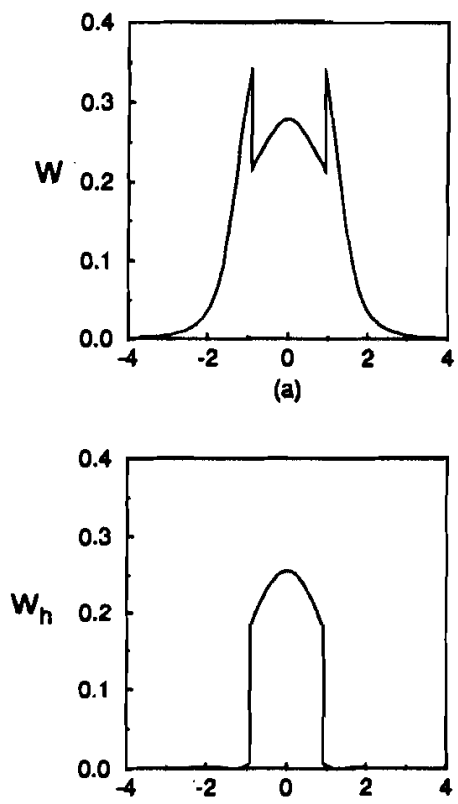

(c) density as a function of position is nearly independent of the particle shape along this direction. However, for the four-fold shapes the deviatoric energy density is quite large in the matrix near the interface and quite low within the particle. In contrast, there is no rapid rise in the deviatoric energy density near the interface of the two-fold particle and a much larger, but nearly uniform, deviatoric energy density within the particle. Thus, the transition from a four-fold to a two-fold shape is driven by the decrease in the large
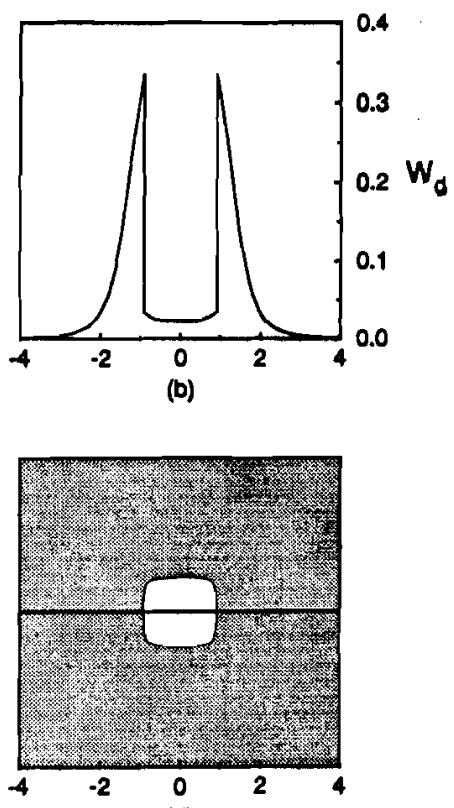

(d)

Fig. 5. Dimensionless (a) elastic energy density, (b) deviatoric energy density, and (c) hydrostatic energy density, as a function of position along the line shown in (d) at $L=10$. 


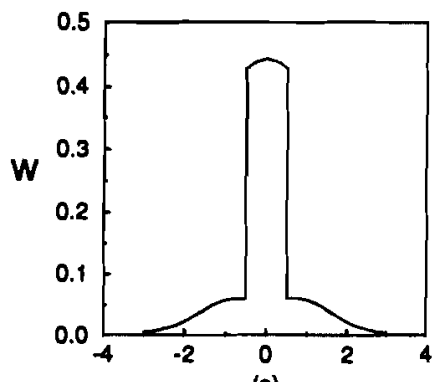

(a)

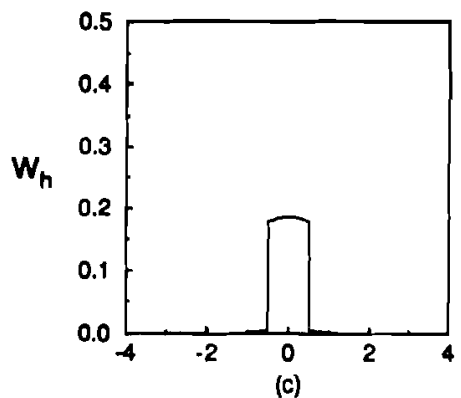

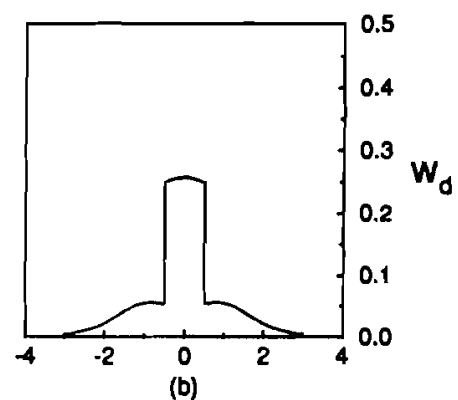

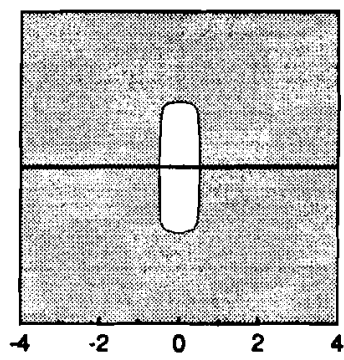

(d)

Fig. 6. Dimensionless (a) elastic energy density, (b) deviatoric energy density, and (c) hydrostatic energy density, as a function of position along the line shown in (d) for $L=10$.

deviatoric energy density in the matrix near the interface of the four-fold particle. As the deviatoric energy density of a fluid at equilibrium is always zero, one would not expect to see such shape transitions in fluids or hydrostatically stressed solids.

\section{2. $A_{r}>1, \epsilon^{r} \neq 1$}

Here we employ the same elastic anisotropy as was used previously, $A_{\mathrm{r}}=2.52$, and add a tetragonal misfit along the $[010]$ direction. We first examine the case of a small tetragonal misfit, $\epsilon^{\mathrm{r}}=1.05$, corresponding to a lattice parameter of the precipitate which is $5 \%$ larger in the [010] direction than in the [100] direction. Even though the degree of tetragonality is small, this changes dramatically the structure of the bifurcation diagram. Figure 7 shows that the addition of a small amount of tetragonality acts as an imperfection to the bifurcation seen previously for a purely dilatational misfit. Now one never observes four-fold symmetric shapes, as the tetragonality introduces a two-fold symmetry for any nonzero value of $L$. For small $L$, the particle is

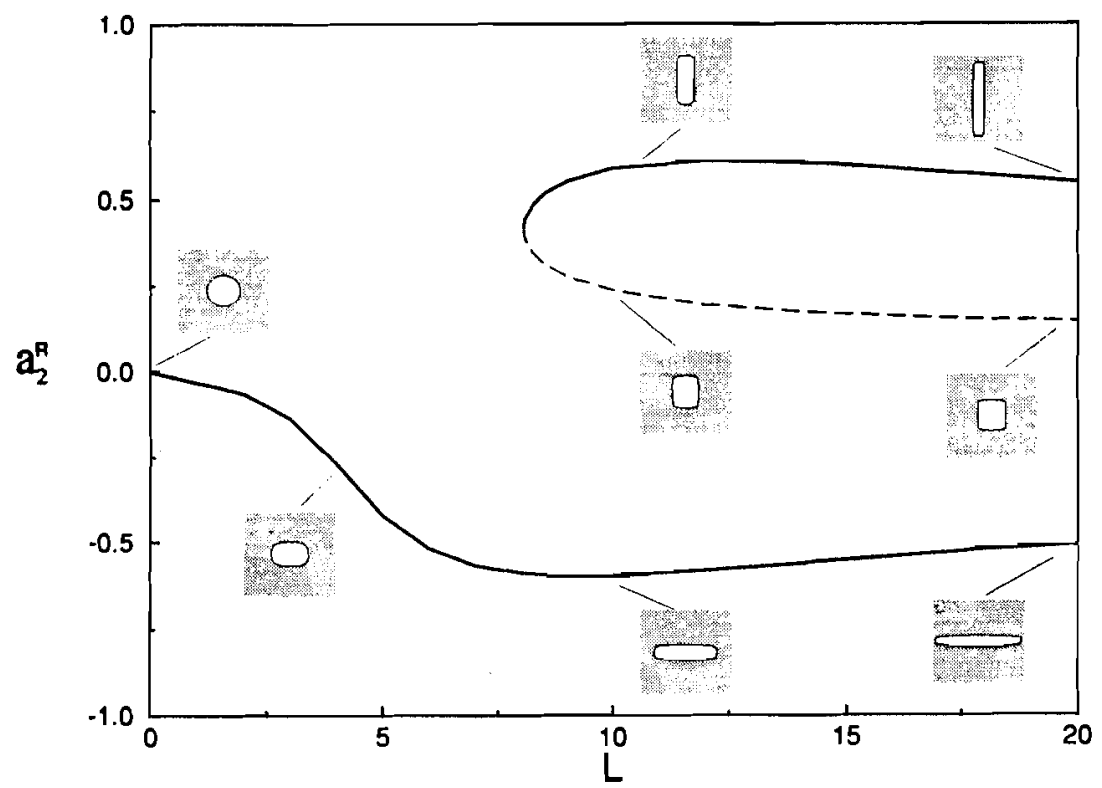

Fig. 7. Bifurcation diagram for a tetragonally misfitting particle with $\epsilon^{\prime}=1.05$. 
elongated in the direction perpendicular to that with the larger misfit, and thus there is a family of particle shapes emanating from the origin with $a_{2}^{\mathrm{R}}<0$. Above $L=8.0$, we find three distinct families of two-fold energy extremizing shapes: particles oriented along [100] and [010], which are energy minima, and a family of energy maximizing shapes which separate these two energy minima. These two families of two-fold energy minimizing shapes are not symmetryrelated variants as the two-fold shape oriented along [010] is a relative energy minimum and the two-fold shape oriented along [100] is an absolute energy minimum. Thus, the family of shapes for which $a_{2}^{\mathrm{R}}<0$ is always the global energy minimum. There is also a turning point in the bifurcation diagram, implying that two-fold shapes oriented parallel to the direction of tetragonality cannot exist below the value of $L$ at the turning point, $L=8.0$.

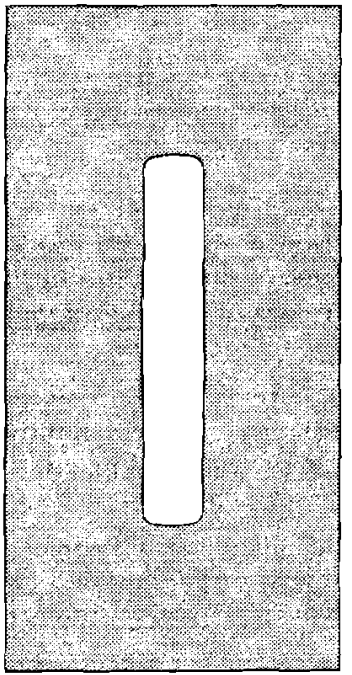

(a)

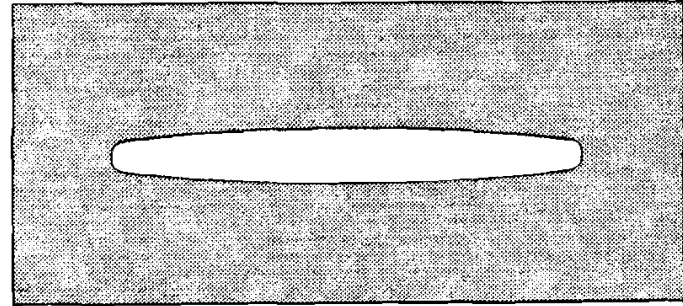

(c)

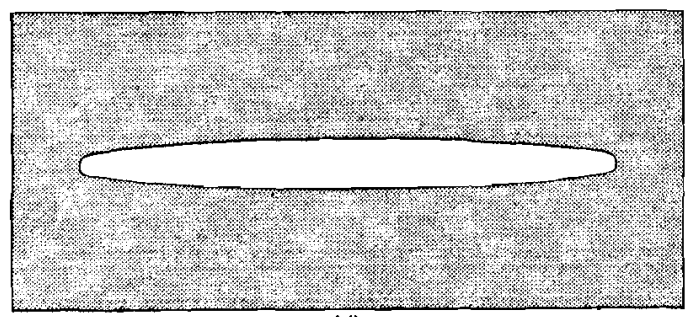

(d)

Fig. 8. Energy minimizing particle shapes for $L=20$ for various values of $\epsilon^{\mathrm{r}}$ : (a) 1.10 , (b) 1.25 , (c) 1.10 , (d) 1.25 . The particle shapes in (a) and (b) are relative energy minima while (c) and (d) are absolute energy minima.
The difference between the two energy minimizing shapes becomes more evident at higher values of tetragonality. To illustrate, we have fixed $L$ at 20 , increased the tetragonality parameter, and determined the energy minimizing particle shapes. This is, in essence, adding a third dimension, $\epsilon^{r}$, to the bifurcation diagram shown in Fig. 7. Figure 8 shows the energy minimizing shapes for various $\epsilon^{\mathrm{r}}$ at $L=20$. The two particle shapes shown in Fig. 8(a) and $8(b)$ correspond to the uppermost family of solutions shown in the bifurcation diagram of Fig. 7 and thus are relative energy minima, whereas the particle shapes shown in Fig. $8(\mathrm{c})$ and $8(\mathrm{~d})$ are the absolute energy minima. The difference in morphology between the energy minimizing particle shapes oriented along [100] and [010] is clear. At $\epsilon^{\mathrm{r}}=1.1$ both particle shapes are convex, with the particle oriented along [010] having flatter sides and ends than the particle oriented along [100]. However, at $\epsilon^{t}=1.25$, the particle oriented along [010] is no longer convex and has large negative curvature along the sides of the particle as shown in Fig. 9. As this hourglass-like particle shape is an energy minimum, it is clear that the equilibrium shape of a misfitting particle can be nonconvex. When $\epsilon^{r} \geq 1.25$, the turning point evident in Fig. 7 is encountered and the solution family of relative energy minimizing shapes merges with the energy maximizing shapes and thus ceases to exist. This implies that at this value of $L$ there can be only one equilibrium shape if the degree of tetragonality exceeds 1.25 . In contrast to the particle shapes which are relative energy minima, particles aligned along [100] become longer and thinner as $\epsilon^{r}$ increases. In addition, the length of the ends of the particles become smaller, and the average curvature of the sides of the particle increases.

\section{3. $A_{r}<1, \epsilon^{r}=1$}

Here we determine the energy extremizing shapes of a particle with a dilatational misfit in a system where the elastically soft directions of the crystal are $\langle 110\rangle$. We have chosen the anisotropy ratio of $\mathrm{ZrO}_{2}$, $A_{\mathrm{r}}=0.4$. We obtain the bifurcation diagram shown in Fig. 10. We now use the value of $a_{2}^{1}$ to characterize a solution family in order to more clearly show the symmetries involved in the particle shape bifurcations. As in the case of $\mathrm{Ni}$, we see both four-fold and two-fold symmetric shapes. The two-fold shapes are again located along the elastically soft directions of the crystal, which in this case are the $\langle 110\rangle$, and the four-fold shapes have sides oriented perpendicular to the $\langle 110\rangle$. However, due to the larger magnitude of the elastic constant anisotropy of $\mathrm{ZrO}_{2}$, the bifurcation point is located at a lower value of $L, L=2.1$, instead of 5.6 as observed for $\mathrm{Ni}$.

\section{4. $A_{r}<1, \epsilon^{r} \neq 1$}

Using the elastic constant anisotropy ratio of $\mathrm{ZrO}_{2}$, when a tetragonal distortion along the [010] direction is added, $\epsilon^{r}=1.37$, the bifurcation diagram 


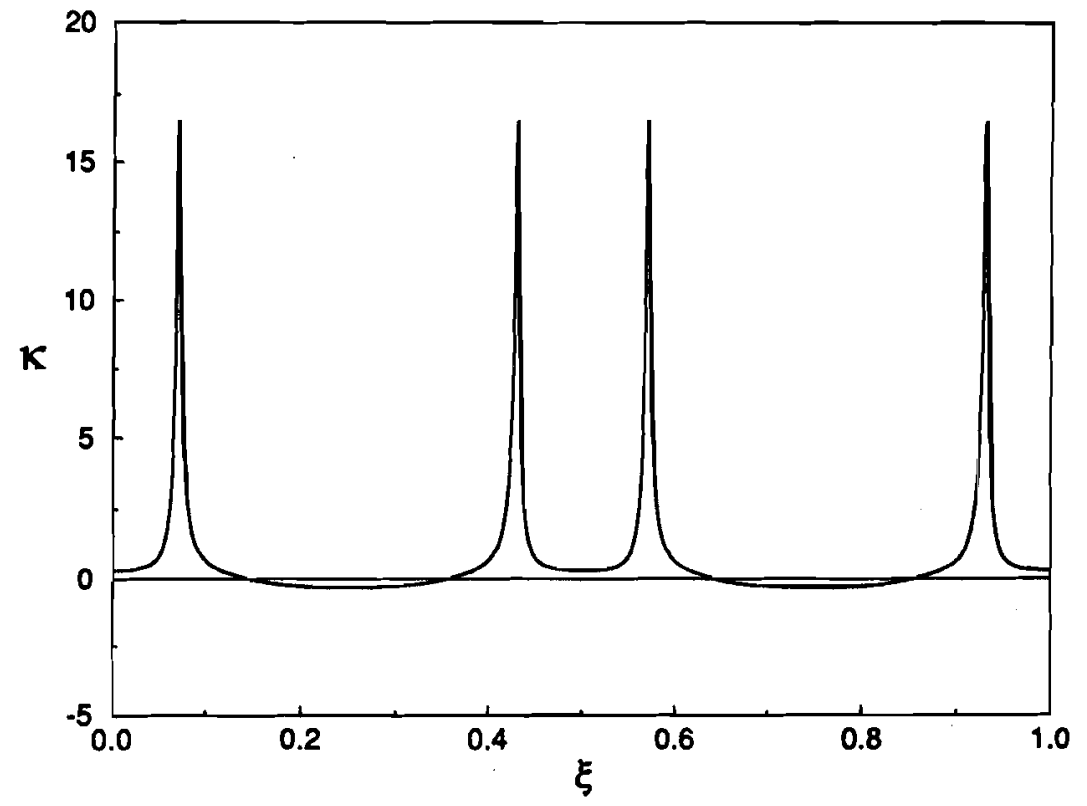

Fig. 9. Interfacial curvature as a function of the relative arc length, $\xi$, for the energy minimizing shape shown in Fig. 8(b).

shown in Fig. 11 is obtained. In contrast to the case where $A_{\mathrm{r}}>1$, the addition of a tetragonal misfit does not break the supercritical bifurcation observed for the case of a dilatational misfit. This is because when $A_{1}<1$, the direction of the tetragonal misfit is not aligned with an elastically soft direction of the crystal. Thus, the presence of a tetragonal misfit does not favor one two-fold variant over the other, as was the case for $A_{r}>1$, and the shape bifurcation remains supercritical. The two energy minimizing particle shapes present above the bifurcation point are symmetry related variants. The presence of a tetragonal misfit, however, does move the location of the bifurcation point to lower values of $L$. A tetragonal misfit also alters the particle shapes from the simple four-fold and two-fold shapes shown in Fig. 10, see Fig. 12. In this case the presence of a tetragonal misfit causes the four-fold shapes shown in Fig. 10 to elongate along [100], as happened previously for $A_{\mathrm{r}}>1$. Rather than taking on plate-like shapes, however, the particles become two-fold diamond-like shapes. In addition, the two-fold shapes shown in

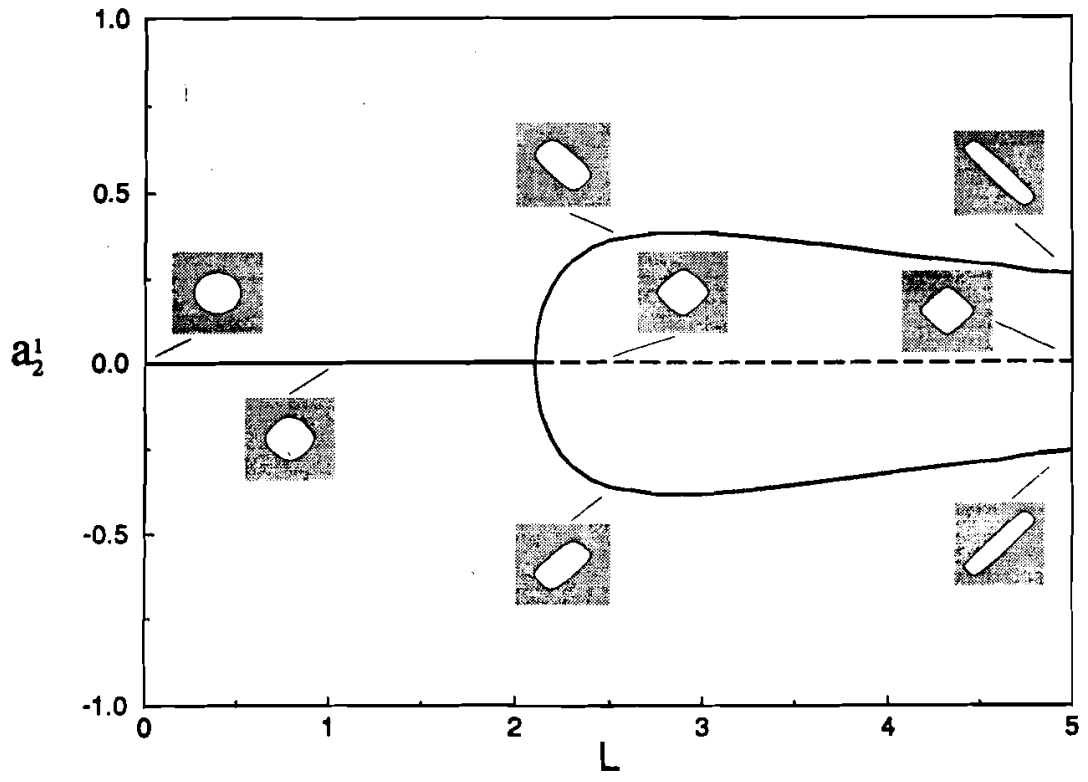

Fig. 10. Bifurcation diagram for dilatationally misfitting particles in a system where the elastically soft directions are the $\langle 1 \mid 0\rangle, A_{\mathrm{r}}=0.4$. 


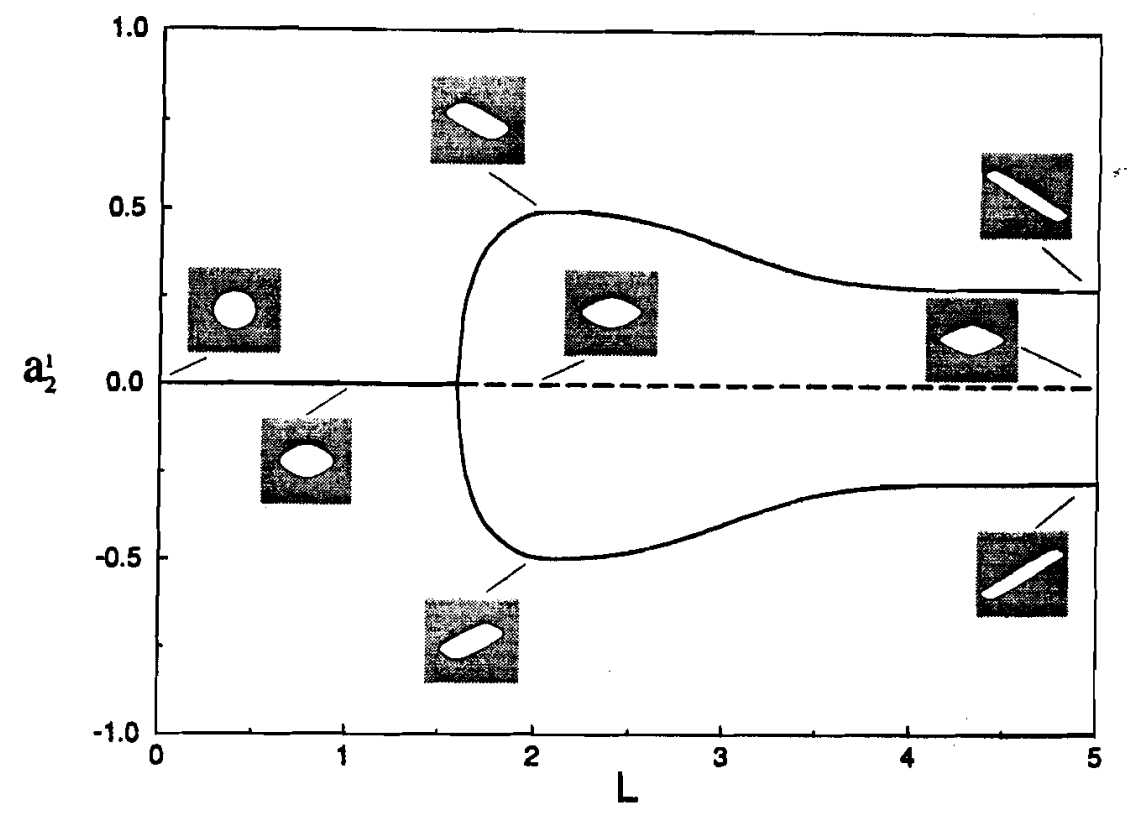

Fig. 11. Bifurcation diagram for $\epsilon^{r}=1.37$ and $A_{r}=0.4$.

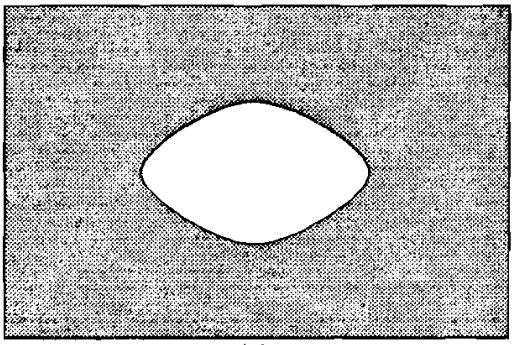

(a)

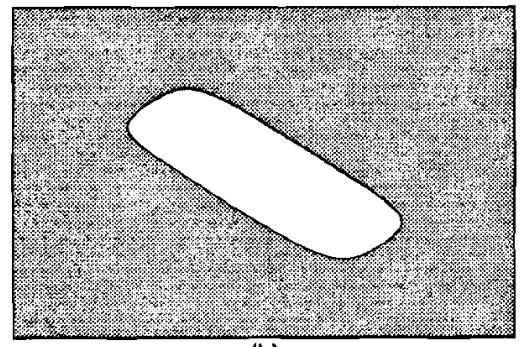

(b)

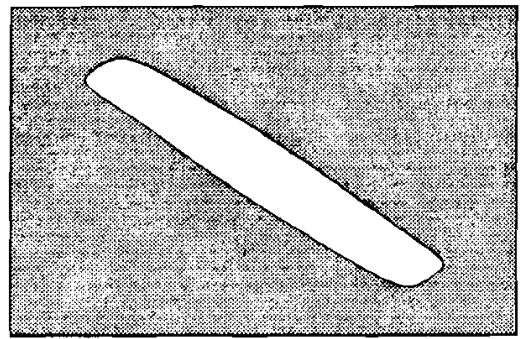

(c)

Fig. 12. Equilibrium particle shapes for $\epsilon^{r}=1.37$ and $A_{r}=0.4$, (a) $L=1.5$ which is below the bifurcation point, (b) $L=2.5$ and (c) $L=5$.
Fig. 11 which exist past the bifurcation point tend to fold over near their ends. This folding is most obvious for the particle with $L=5$, Fig. 12(c), where there is a small region of negative curvature near the ends of the particle. This folding is due to the competition between the tetragonality driving the particle to elongate along [100] and the elastic constant anisotropy which forces the particle to elongate along $\langle 110\rangle$. Since these S-like shaped particles appear above the bifurcation point, they are energy minima.

\section{DISCUSSION}

We have shown that there is a marked difference between the equilibrium particle shape problem in the absence of elastic stress and that in the presence of stress. The equation defining the equilibrium shape changes from a purely differential equation for $L=0$ to an integrodifferential equation for $L \neq 0$, see equation (3.10). The integral nature of the defining equation reflects the long-ranged nature of the elastic stress; it is not possible to determine the value of the diffusion potential at a point on the interface until the entire shape of the particle is known. In contrast to the classical surface energy minimizing shapes, we find that the equilibrium shape is a function of the particle size, see also $[18,21]$, can be nonunique for a given set of thermophysical parameters, and can be nonconvex. The presence of such nonconvex equilibrium shapes illustrates the importance of not constraining the particles to be of a certain shape when determining the equilibrium morphologies of misfitting particles.

Depending on the parameters, we find that supercritical particle-shape bifurcations are possible. 
Supercritical particle-shape bifurcations have been found previously by assuming that the particle morphology is in a class of simple geometric shapes $[14,15]$. While it is encouraging that the presence of these supercritical bifurcations is not destroyed when considering particles with an equilibrium shape, it is not clear that such qualitative similarities between constrained and unconstrained particle shape calculations will exist in other situations. These supercritical bifurcations, or second-order transitions in phase transition language, imply a continuous change in particle morphology from a four-fold shape to a two-fold shape as $L$ is varied. In addition, the presence of such a bifurcation implies that the four-fold family of shapes is unstable above the bifurcation point and there is no metastability.

When the tetragonal misfit is aligned along one of the elastically soft directions of the crystal, the supercritical bifurcation present for a dilatational misfit is broken. The broken bifurcation shown in Fig. 7 implies that a particle can undergo a discontinuous change in shape with a change in $L$. Consider the evolution of a two-fold symmetric particle oriented in the [010] direction with a value of $L$ exceeding 8 . According to the bifurcation diagram if this particle were to shrink, as might happen during Ostwald ripening, the aspect ratio of the particle would decrease continuously until the turning point is reached, at which point the particle's morphology would change discontinuously to a two-fold shape aligned along the [100] direction. In contrast, a particle which is growing from $L=0$ may simply move through the lower family of particle shapes shown in Fig. 7 and elongate along the [100]. In addition, the presence of a tetragonal misfit introduces metastable states, or relative energy minima. Thus, there can be more than one equilibrium particle shape for a given particle size and set of materials parameters.

Many of the particles shown in the figures exhibit negative interfacial curvature. However, in some of these cases such particles are unstable to other shapes. For example, in a system with a dilatational misfit for $L \gtrless 25$ the four-fold shapes shown in Fig. 1 with negative interfacial curvature are unstable to the formation of particles with two-fold shapes. Nevertheless, we show two examples where the equilibrium shapes can be nonconvex, see Fig. 8 and Fig. 12 . Misfitting particles with negative interfacial curvature have been observed experimentally in the $\mathrm{Ni}-\mathrm{Al}$ system $[38,39]$ and in the $\mathrm{Mg}$-stabilized $\mathrm{ZrO}_{2}$ system [40]. Our results are inconsistent with the experiments in the $\mathrm{Ni}-\mathrm{Al}$ system for we predict that four-fold symmetric particles with negative interfacial curvature should be unstable with respect to the two-fold symmetric particles. This inconsistency may be due to the plane strain nature of our calculations. In contrast, our calculated particle shapes are qualitatively consistent with the $S$-shaped particles observed experimentally in $\mathrm{Mg}$-stabilized $\mathrm{ZrO}_{2}$ [40], as well as those calculated in two-dimensions [41]. Thus, we suggest that these unusual S-shaped particles observed experimentally may nearly be equilibrium shapes, and not the result of multiparticle diffusional and elastic interactions. However, the quantitative difference between the particle shapes we calculate and those observed by Batemen and Notis and determined theoretically by Wang and Katchaturyan is likely due to the comparatively small value of $\epsilon^{\prime}$ used in our work. In $\mathrm{Mg}$-stabilized $\mathrm{ZrO}_{2}, \epsilon^{r}=34$ instead of the 1.37 used herein.

It is clear from the experiments of Miyazaki et al. that elastic stress can induce a particle to split into smaller particles [39]. However, the method by which a particle splits is a matter of some controversy. Miyazaki suggested that a particle splits by the formation of the matrix phase within the particle. In contrast, the experiments of Kaufman et al. seem to suggest that the particle splits by the growth of interfacial perturbations. The recent experiments by Yoo and Yoon [42], in which the three-dimensional nature of the splitting process can be observed, support the idea that the splitting process begins by the amplification of interfacial perturbations. Theoretically, particle splitting by matrix phase nucleation was found to occur in the time dependent calculations of both Chen et al. [18] and McCormick et al. [20]. While our calculations will not permit the nucleation of the matrix phase within the particle, our results appear to contradict those of Chen et al., as they find that four-fold symmetric shapes with negative curvature are metastable and, past a critical particle size, are unstable to the formation of matrix phase within the particle. In contrast, we find that such four-fold particles with negative curvature are unstable to the formation of two-fold shapes. However, our results are qualitatively consistent with the energetic calculations of McCormick et al., where they predict that a plate is an energy minimum at smaller particle sizes than a square. Potentially important differences between our work and that of Chen et al. are that we do not allow the lattice parameter to vary with composition and thus their equilibrium particle shapes satisfy a different variational principle from the one shown in equation (3.2) [27], and we constrain the elastic field to be one of plane strain whereas they assume it to be twodimensional. It remains for future work to address the causes of the differences in our calculations. Nevertheless, since we find that the four-fold symmetric particles are stable to interfacial perturbations which would lead to splitting, at least to $L=26$, the splitting process observed experimentally may be an inherently three-dimensional effect which is not describable within the context of a plane strain calculation.

Accurate techniques for measuring interfacial energies often balance the effects of capillarity against an external field. For example, when the gravitationally induced pressure gradient in a fluid is balanced by the 
pressure jump across a curved interface, the interfacial energy of a two-phase fluid interface can be measured by the well-known capillary rise or sessile drop technique. In solid-liquid systems, when the curvature dependence of the interfacial temperature is balanced against an applied temperature gradient, the profile of a grain boundary groove can yield an accurate measurement of the solid-liquid interfacial energy [43]. One factor leading to the success of both of these techniques is that these two-phase systems are at equilibrium. Unfortunately, such approaches are not possible in solid-solid systems, and thus in most cases interfacial energies of coherent interfaces have been determined through recourse to kinetic, and not equilibrium, measurements. Extracting accurate measurements of interfacial energies from, for example, measured coarsening kinetics can be quite difficult [44]. However, a field against which the effects of interfacial energy can be balanced in a solid-solid system is the elastic stress field due to a particle-matrix misfit. Our calculations suggest that if it is possible to find a match between an experimentally measured particle shape and that calculated theoretically, one can deduce the value of $L$. Since the other thermophysical parameters appearing in $L$ are usually known and the size of the particle can be determined, the value of the interfacial energy can be found from the expression for $L$. Another approach is to determine experimentally the particle size at which a supercritical shape bifurcation occurs. With knowledge of the experimentally measured critical particle size and the theoretically determined critical value of $L$ at which the shape bifurcation should occur, one can again solve for the interfacial energy. However, there are some important factors which need to be considered: the particle of interest must be sufficiently isolated from the other particles in the system so that interparticle elastic and diffusional interactions are not important and the theoretical calculations must be three-dimensional. Of these, the most difficult to achieve may be the first, as shown by the recent results of Maheshwari and Ardell [45].

\section{CONCLUSIONS}

We examined the equilibrium morphologies of precipitates with either a tetragonal or purely dilatational misfit in an elastically anisotropic medium with cubic symmetry under conditions of plane strain. We find that:

1. For a system with a purely dilatational misfit, the equilibrium particle morphology changes smoothly from a circle to a four-fold symmetric shape and, at a critical value of $L$, bifurcates supercritically to two-fold symmetric shapes aligned along the elastically soft directions of the crystal. In addition, we find that all the four-fold symmetric shapes are stable with respect to interfacial perturbations which would lead to the experimentally observed particle splitting.

2. The presence of a tetragonal misfit acts as an imperfection to this supercritical bifurcation when the direction of tetragonality coincides with an elastically soft direction of the crystal. At small values of $L$ the tetragonal misfit causes the particle to elongate in a direction perpendicular to the tetragonality. At larger values of $L$ equilibrium particle shapes exist which are local energy minima, or metastable, and may also be strongly nonconvex.

3. When the tetragonal misfit is not aligned along the elastically soft direction, the supercritical bifurcation reappears. Below the bifurcation point the particles take on a two-fold symmetric diamond-like appearance whereas above the bifurcation point the particles are S-shaped,

4. It may be possible to determine the interfacial energy of a coherent interface by measuring the shape of a misfitting particle, or the critical particle size at which a particle shape bifurcation occurs.

Acknowledgements-We acknowledge many helpful discussions with G. B. McFadden and D. N. Yoon. In addition, we are most grateful for discussions with $Y$. Wang and A. G. Khachaturyan, and, in particular, for sharing with us the results of their calculations on the evolution of $\mathrm{t}-\mathrm{ZrO}$ precipitates prior to publication. We thank the National Science Foundation, DMR-8957219, for financial support.

\section{REFERENCES}

1. J. E. Taylor, Bull. Am. Math. Soc. 84, 197 (1978).

2. I. Fonseca, Proc. R. Soc. A 432, 125 (1991).

3. W. W. Mullins, J. Math. Phys. 3, 754 (1962).

4. C. A. Johnson and G. D. Chakerian, J. Math. Phys. 6, 1403 (1965)

5. J. K. Lee, H. I. Aaronson and K. C. Russell, Surf. Sci. 51, 302 (1975).

6. E. Arbel and J. W. Cahn, Surf. Sci. 51, 305 (1975).

7. W. C. Johnson and P. W. Voorhees, Solid St. Phenom. 23, 87 (1992).

8. T. Miyazaki and M. Doi, Mater. Sci. Engng a110, 175 (1989).

9. A. G. Khachaturyan, S. V. Semenovskaya and J. W. Morris, Acta metall. 36, 1563 (1988).

10. A. G. Khachaturyan, Theory of Structural Phase Transformations in Solids. Wiley, New York (1983).

11. A. G. Khachaturyan and G. A. Shatalov, Soviet Phys. Solid St. 11, 118 (1969).

12. I. M. Kaganova and A. L. Roitburd, Soviet Phys. JETP 67,1173 (1988).

13. W. C. Johnson and P. W. Voorhees, J. appl. Phys. 61, 1610 (1987).

14. W. C. Johnson and J. W. Cahn, Acta metall. 23, 1839 (1984).

15. W. C. Johnson, M. B. Berkenpas, and D. E. Laughlin, Acta metall. 36, 3149 (1988).

16. M. A. Grinfeld, Phys. Earth Planetary Interiors 50, 99 (1988).

17. A. Onuki, J. Phys. Soc, Japan 58, 3069 (1989).

18. Y. Wang, L. Q. Chen and A. Khachaturyan, Acta metall. mater. 41, 279 (1993).

19. J. K. Lee, Metall. Trans. 22A, 1197 (1991). 
20. M. McCormack, A. G. Khachaturyan and J. W. Morris, Acta metall. mater. 40, 325 (1992).

21. P. W. Voorhees, G. B. McFadden and W. C. Johnson, Acta metall. mater. 40, 2979 (1992).

22. F. C. Larché and J. W. Cahn, Acta metall. 21, 1051 (1973).

23. F. C. Larché and J. W. Cahn, Acta metall. 26, 1579 (1978).

24. W. C. Johnson and J. I. D. Alexander, J. appl. Phys. 59, 2735 (1986).

25. P. H. Leo and R. F. Sekerka, Acta metall. 37, 3119 (1989).

26. A. L. Roitburd, Soviet Phys. Dokald. 16, 305 (1971).

27. M. E. Gurtin and P. W. Voorhees, Proc. Roy. Soc. A 440, 323 (1993).

28. D. W. Hoffman and J. W. Cahn, Surf. Sci. 31, 368 (1972).

29. J. W. Cahn and D. W. Hoffman, Acta metall. 22, 1205 (1974).

30. T. Mura, Micromechanics of Defects in Solids. Martinus Nijhoff, The Hague (1982).

31. D. J. Bacon, D. M. Barnett and R. O. Scattergood, Prog. Mater, Sci. 23, 51 (1979)

32. A. J. Ardell and R. B. Nicholson, Acta metall. 14, 1295 (1966).
33. L. N. Brush, G. B. McFadden and S. R. Coriell, J. Cryst. Growth 114, 446 (1991).

34. G. B. McFadden, P. W. Voorhees, R. F. Boisvert and D. I. Meiron, J. Sci. Comput. 1, 117 (1986)

35. A. Sidi and M. Israeli, J. Sci. Comput. 3, 201 (1988).

36. G. Baker and A. Nachbin, J. Comp. Phys. To be published.

37. A. G. Khachaturyan, Soviet Phys. Solid St. 8, 2163 (1967).

38. A. Maheshwari and A. J. Ardell, Scripta metall. mater. 26, 347 (1992)

39. T. Miyazaki, H. Imamura and T. Kozakai, Mater. Sci. Engng 54, 9 (1982).

40. C. A. Bateman and M. R. Notis, Acta metall. 40, 2413 (1992).

41. Y. Wang and A. G. Khachaturyan, unpublished.

42. Y. S. Yoo, Ph.D. dissertation, Korean Advanced Inst. of Sci. Technol. (1992).

43. R. J. Schaefer, M. E. Glicksman and J. D. Ayers, Phil. Mag. 32, 725 (1975).

44. H. A. Calderon, P. W. Voorhees, J. L. Murray and G. Kostorz, Acta metall. mater. 42, 991 (1994).

45. A. Maheshwari and A. J. Ardell, Phys. Rev. Lett. 70, 2305 (1993) 